\title{
SENSORIAMENTO REMOTO NA DETERMINAÇÃO DE ATRIBUTOS DE UM NITOSSOLO SOB APLICAÇÃO DE VINHAÇA $^{(1)}$
}

\author{
Juliano Araujo Martins ${ }^{(2)}$, Peterson Ricardo Fiorio ${ }^{(3)}$, José A. M. Demattê( ${ }^{(4)}$, Jarbas \\ Honorio de Miranda ${ }^{(5)}$ \& João Alberto Lelis Neto ${ }^{(6)}$
}

RESUMO

\begin{abstract}
O sensoriamento remoto vem sendo utilizado na avaliação de características químicas e físicas dos solos, como fonte de informação rápida, não destrutiva e de baixo custo, podendo assim auxiliar no gerenciamento de passivos ambientais. Nesse sentido, este trabalho teve por objetivo verificar o potencial da utilização de dados hiperespectrais na determinação de algumas propriedades de um solo submetido a diferentes aplicações de doses de vinhaça, utilizando dados espectrais oriundos de amostras em condições de campo e de terra fina seca em estufa. $O$ experimento, delineado em blocos ao acaso constou de seis tratamentos e quatro repetições, sendo os tratamentos: 1, sem vinhaça; 2 , fertirrigado com químicos; e 3, 4, 5 e 6, com aplicação de, respectivamente, $150,300,600$ e $900 \mathrm{~m}^{3} \mathrm{ha}^{-1}$ de vinhaça. Foram gerados modelos para predição de alguns atributos químicos e físicos do solo para os dois tipos de amostras, a partir de curvas espectrais na região do visível e infravermelho próximo. Para determinação da granulometria, os modelos não foram influenciados pelo tipo de amostra utilizada e foram classificados como "aceitáveis" a "bons" $\left(R^{2}\right.$ entre $0,7 \mathrm{e}$ $0,9)$. Em relação aos atributos químicos, foram gerados modelos com capacidade de diferenciação apenas entre altas e baixas concentrações $\left(R^{2}\right.$ entre 0,50 e 0,65$)$ para os atributos pH, matéria orgânica, $\mathrm{H}+\mathrm{Al}$ e capacidade de troca catiônica,
\end{abstract}

(1) Parte da Dissertação de Mestrado do primeiro autor, apresentada ao PPG em Irrigação e Drenagem da Escola Superior de Agricultura Luiz de Queiroz - ESALQ/USP. Recebido para a publicação em 14 de fevereiro de 2013 e aprovado em 24 de fevereiro de 2014 .

(2) Professor Mestre, Instituto Federal de Mato Grosso, Campus Sorriso. Av. Tancredo Neves, 543. CEP 78.890-000 Sorriso (MT). E-mail: julianoaraujo3@gmail.com

(3) Professor Doutor, Departamento de Engenharia de Biossistemas, ESALQ/USP. E-mail: fiorio@usp.br

(4) Professor Livre Docente-3, Departamento de Ciência do Solo, ESALQ/USP. E-mail: jamdemat@usp.br

(5) Professor Associado-2, Departamento de Engenharia de Biossistemas, ESALQ/USP. E-mail: jhmirand@usp.br

(6) Doutor em Irrigação e Drenagem, ESALQ/USP. E-mail: joao_lelis@yahoo.com.br 


\begin{abstract}
sendo os gerados para terra fina seca em estufa em alguns casos melhores que os para amostras em condições de campo.
\end{abstract}

\author{
Termos de indexação: espectrorradiometria, análise multivariada, propriedades \\ do solo.
}

\title{
SUMMARY: REMOTE SENSING TO DETERMINE THE PROPERTIES OF AN ALFISOL UNDER SUGARCANE RESIDUE APPLICATION
}

\begin{abstract}
Remote sensing has been used in the evaluation of physical and chemical characteristics of soils as a source of rapid, non-destructive, and low cost information, which may assist in management of environmental liabilities. The aim of this study was to investigate the potential for use of hyperspectral data in determining some properties of soil under different levels of application of sugarcane residue (vinasse) using spectral data obtained from samples in field conditions and laboratory oven-dried soil. A randomized block design was used with six treatments and four replications, the treatments being: 1. without vinasse; 2. fertigation with chemicals; 3, 4, 5, and 6 received the application of 150, 300,600, and $900 \mathrm{~m}^{3} \mathrm{ha}^{-1}$ of vinasse, respectively. Models were generated for prediction of chemical and physical soil properties for both types of samples from spectral curves of visible and near-infrared wavelengths. For determination of soil particle size, models classified as acceptable to good ( $R^{2}$ from 0.7 to 0.9 ) were generated, and these models were not affected by the type of sample used. In regard to chemical properties, models were generated with capacity only for differentiation between high and low concentrations ( $R^{2}$ from 0.5 to 0.65 ) for the properties of $p H$, organic matter, $H+A l$ and cation exchange capacity. The models generated for laboratory oven-dried soil were in some cases better than those generated for samples in field conditions.
\end{abstract}

Index terms: spectroradiometry, multivariate analysis, soil properties.

\section{INTRODUÇÃO}

A vinhaça é um dos principais subprodutos da agroindústria canavieira, caracterizada como efluente de alto poder poluente e também por possuir alto valor fertilizante. Esse produto vem sendo utilizado desde a década de 1980, mas o controle de sua aplicação em lavouras de cana-de-açúcar iniciou-se apenas nos últimos anos (Silva et al., 2007). Visando racionalizar essa utilização, a Companhia de Tecnologia de Saneamento Ambiental (CETESB) criou uma norma técnica que regulamenta a aplicação da vinhaça no Estado de São Paulo (CETESB, 2005).

As alterações dos atributos do solo provenientes da aplicação da vinhaça ao longo do tempo podem ser avaliadas com a utilização de análises de laboratório rotineiras, o que, para fins de monitoramento de grandes áreas, acaba tornando-se inviável em razão principalmente do custo e tempo despendidos para execução dos métodos. Nesse sentido, técnicas alternativas para avaliação das condições químicas e físicas dos solos vêm sendo estudadas. Dentre essas, o sensoriamento remoto (SR) tem recebido grande atenção por parte dos pesquisadores, principalmente por se tratar de uma técnica pouco destrutiva, de ágil verificação e processamento dos resultados, permitindo avaliar vários atributos simultaneamente sem a geração de poluentes em potencial (Dunn et al., 2002; Brown et al., 2006), possibilitando, ainda, seu uso tanto em laboratório quanto "in loco" (Viscarra Rossel et al., 2009).
Estudos referentes à espectroscopia de reflectância difusa utilizando regiões do visível e infravermelho próximo (Vis-NIR) na área de ciência do solo têm aumentado rapidamente nos últimos 10 anos. O principal foco dessas investigações tem sido principalmente os componentes básicos do solo, como matéria orgânica, atributos químicos, umidade, mineralogia da fração argila, entre outros.

Vários trabalhos têm avaliado as variações químicas e físicas dos solos e sua relação com as alterações na resposta espectral desses (Demattê \& Garcia, 1999; Chang et al., 2001; Lee et al., 2003; Demattê et al., 2010; Fiorio \& Demattê, 2009; Viscarra Rossel et al., 2010), alcançando bons resultados, que têm auxiliado sobretudo em levantamentos pedológicos, manejo da fertilidade e conservação dos solos.

Segundo Cohen et al. (2007), é possível a predição de atributos do solo com precisão igual a de resultados de análises convencionais. No entanto, não há dúvidas que variações quanto à classe de solo, à faixa do espectro avaliada, às condições das amostras com relação à umidade, à rugosidade e aos atributos envolvidos na análise exercem grande influência na geração e validação de um modelo universal; sendo assim, estudos localizados para verificação do potencial dessa ferramenta são de grande importância.

O objetivo desta pesquisa foi avaliar a possibilidade da utilização de técnicas de sensoriamento remoto para a quantificação de algumas propriedades do solo, submetido à aplicação da vinhaça, visando auxiliar 
tanto no gerenciamento agrícola quanto no monitoramento de passivos ambientais. A hipótese formulada foi a de que os modelos para predição da granulometria poderiam sofrer interferência pela adição de vinhaça, sobretudo os gerados para amostras em condição de campo, que também poderiam ser influenciados por outros fatores como umidade e rugosidade das amostras.

Para responder a essa questão, foram gerados modelos de regressão por mínimos quadrados parciais a partir de curvas espectrais do solo, para determinação de alguns atributos químicos e físicos, utilizando-se amostras de terra fina seca em estufa e amostras em condição de campo, comparando o desempenho desses modelos com métodos consagrados de análises laboratoriais.

\section{MATERIAL E MÉTODOS}

\section{Área experimental}

O experimento foi conduzido na Fazenda Areão pertencente à Escola Superior de Agricultura "Luiz de Queiroz" (ESALQ/USP), na área experimental do Departamento de Engenharia Biossistemas, município de Piracicaba, SP, $22^{\circ} 41^{\prime} 55^{\prime}$ S, $47^{\circ} 38^{\prime} 41^{\prime}$ 'W e altitude de $511 \mathrm{~m}$. O clima da região é classificado no sistema Köppen como Cwa, denominado subtropical úmido, com pluviosidade anual em torno de $1.257 \mathrm{~mm}$, sendo o período seco o inverno; as médias de temperatura são $22^{\circ} \mathrm{C}$, no mês mais quente, e $18{ }^{\circ} \mathrm{C}$, no mês mais frio, com média anual de $21,4^{\circ} \mathrm{C}$. O solo estudado foi um Nitossolo Vermelho eutroférrico (NVef), de acordo com a classificação brasileira de solos (Embrapa, 2006), cujas características químicas antes da aplicação dos tratamentos são apresentadas no quadro 1.

A área experimental possuía 24 parcelas, de cinco linhas e $8 \mathrm{~m}$ de comprimento com espaçamento de 1,5 m nas entrelinhas. Dessa forma, a área de cada parcela foi de $88 \mathrm{~m}^{2}$, sendo a área total do experimento de $2.112 \mathrm{~m}^{2}$. Para área útil, onde foram realizadas as coletas de solos, consideraram-se as três linhas centrais, descartando $1 \mathrm{~m}$ nas extremidades de cada parcela; logo, cada parcela tinha $24 \mathrm{~m}^{2}$ de área útil.

$\mathrm{O}$ arranjo experimental foi em blocos casualizados, com seis tratamentos e quatro repetições, sendo esses: Tratamento 1, sem aplicação de vinhaça, com adubação segundo as recomendações para a cultura, realizada no momento do plantio; Tratamento 2 , fertirrigado com fertilizantes químicos, de acordo com a marcha de absorção de nutrientes da cultura; Tratamento 3 , fertirrigado com $50 \%$ da dose de vinhaça recomendada para esse solo, de acordo com as normas técnicas da CETESB; Tratamento 4, fertirrigado com a dose de vinhaça recomendada pela CETESB; Tratamento 5 , fertirrigado com duas vezes a dose de vinhaça recomendada pela CETESB; e Tratamento 6 , fertirrigado com três vezes a dose de vinhaça recomendada pela CETESB.

Com base nas características químicas do solo (Quadro 1), e na concentração de $\mathrm{K}^{+}$na vinhaça, as doses a serem aplicadas foram definidas de acordo com a equação 1 (CETESB, 2005):

$\mathrm{m}^{3} \mathrm{ha}^{-1}$ de vinhaça $=\frac{[(0,05 \times \mathrm{CTC}-\mathrm{Ks}) \times 3744+185]}{\mathrm{Kvi}}$

em que: CTC $=$ Capacidade de Troca Catiônica, expressa em $\mathrm{cmol}_{\mathrm{c}} \mathrm{dm}^{-3}$, a pH 7,$0 ; \mathrm{Ks}=$ concentração de $\mathrm{K}$ no solo, expresso em $\mathrm{cmol}_{\mathrm{c}} \mathrm{dm}^{-3}$, à profundidade de $0,80 \mathrm{~m} ; 3744=$ constante para transformar os resultados da análise de fertilidade, expressos em $\mathrm{cmol}_{\mathrm{c}} \mathrm{dm}^{-3}$, para $\mathrm{kg}$ de $\mathrm{K}$ em volume de 1 ha a $0,80 \mathrm{~m}$ de profundidade; $185=\mathrm{kg}$ de $\mathrm{K}_{2} \mathrm{O}$ médio extraído pela cultura por ha, por corte; e Kvi = concentração de $\mathrm{K}$ na vinhaça, expressa em $\mathrm{kg} \mathrm{m}^{-3} \mathrm{de}_{2} \mathrm{O}$.

Como há grande variação da composição química da vinhaça, foi considerado o resultado médio das análises de cinco amostragens; a concentração de K utilizada para o cálculo da dose recomendada para aplicação foi de $1,91 \mathrm{~g} \mathrm{~L}^{-1}$. As quantidades reais aplicadas ao solo foram de 150, 300, 600, e $900 \mathrm{~m}^{3} \mathrm{ha}^{-1}$ de vinhaça para os tratamentos $3,4,5$ e 6 , respectivamente.

\section{Coleta de solo e análises químicas e físicas}

Foram coletadas amostras deformadas de solo, utilizando-se trado holandês, sendo uma amostragem simples por parcela do experimento, com seis períodos de coleta. A primeira coleta foi feita antes da aplicação dos tratamentos; da segunda à quinta coletas, foram realizadas $24 \mathrm{~h}$ após cada aplicação da fertirrigação convencional e doses de vinhaça; e fez-se a sexta coleta dois meses após o término das aplicações, nas seguintes datas: 21/06/10,19/08/10, 20/10/10, 15/12/ $10,20 / 02 / 11$ e 18/04/11. Realizaram-se as amostragens nas camadas 0,0-0,20 e 0,20-0,40 m.

Os atributos químicos e físicos avaliados foram: $\mathrm{pH}\left(\mathrm{CaCl}_{2}\right)$, teor de matéria orgânica (MO), $\mathrm{P}, \mathrm{K}, \mathrm{Mg}$, $\mathrm{Ca}$ e H+Al. Posteriormente, realizaram-se os cálculos para soma de bases (SB), capacidade de troca catiônica em pH $7\left(\mathrm{CTC}_{\mathrm{pH}}\right)$ e saturação por bases (V\%) (Raij \& Quaggio, 1983) e teores de areia, silte e argila pelo método do densímetro (Camargo et al., 1986).

\section{Obtenção das curvas espectrais}

Foram obtidos dados espectrais para dois tipos distintos de amostras: Amostras em condição de campo (ACC), mantendo-se as condições de umidade do momento da coleta, destorroada, mas sem peneiramento; e Terra fina seca em estufa (TFSE), em que o solo foi seco em estufa a $45{ }^{\circ} \mathrm{C}$ por $24 \mathrm{~h}$ e peneirado em malha de $2 \mathrm{~mm}$, procedimento usualmente utilizado em espectroscopia (Henderson et al., 1992). Ambos os tipos de amostras foram 
Quadro 1. Características químicas prévias do solo da área experimental

\begin{tabular}{|c|c|c|c|c|c|c|c|c|c|c|c|c|c|c|}
\hline Prof. & Argila & Silte & Areia & $\mathrm{pH}\left(\mathrm{CaCl}_{2}\right)$ & MO & $\mathbf{P}$ & $\mathbf{K}$ & $\mathrm{Ca}$ & $\mathrm{Mg}$ & $\mathrm{H}+\mathrm{Al}$ & $\mathrm{Al}$ & SB & $\mathbf{C T C}_{\mathrm{pH} 7}$ & V \\
\hline $\mathrm{m}$ & - & $-\mathrm{g} \mathrm{kg}^{-1}$ & 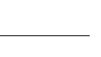 & & $\mathrm{g} \mathrm{kg}^{-1}$ & $\mathrm{mg} \mathrm{dm} \mathrm{m}^{-3}$ & & & & $\mathrm{~mol}_{\mathrm{c}} \mathrm{dn}$ & & & & $\%$ \\
\hline $0,0-0,2$ & 464,0 & 270,2 & 265,8 & 5,0 & 27 & 8 & 2,5 & 30 & 15 & 39 & 1 & 48 & 86 & 55 \\
\hline $0,2-0,4$ & 484,3 & 274,1 & 241,6 & 5,2 & 20 & 5 & 1,7 & 28 & 14 & 33 & 0 & 43 & 77 & 57 \\
\hline
\end{tabular}

acondicionados em placas de "petri” com diâmetro de $9 \mathrm{~cm}$, formando uma camada com espessura de aproximadamente $1,5 \mathrm{~cm}$. Os dados espectrais foram obtidos em laboratório com o espectrômetro FieldSpec Pro (Analytical Spectral Devices, Boulder, Colo). Trata-se de um espectrorradiômetro com fibra ótica possuindo resolução espectral de $1 \mathrm{~nm}$, na faixa espectral de 350 a $1.100 \mathrm{~nm}$, e $2 \mathrm{~nm}$, de 1.100 a $2.500 \mathrm{~nm}$.

O sensor foi alocado na vertical, a $27 \mathrm{~cm}$ de altura das amostras de solo. A fonte de iluminação utilizada foi uma lâmpada halógena de $650 \mathrm{~W}$, com feixe não colimado para o plano visado, posicionada a $35 \mathrm{~cm}$ do recipiente amostral, com um ângulo zenital de $20^{\circ}$. Como referência, utilizou-se uma placa branca com superfície de sulfato de bário considerada como padrão de 100 \% de reflectância. Dessa forma, a razão entre o fluxo radiante espectral refletido pela superfície de uma amostra de solo e o fluxo radiante espectral refletido por um padrão de referência, iluminados e visados sob as mesmas condições geométricas, gerou o "fator de reflectância espectral bidirecional".

A partir do fator de reflectância bidirecional dos solos, obtiveram-se as curvas espectrais. Para cada amostra analisada, foram efetuadas três leituras em diferentes posições da placa, de modo a aumentar a área analisada, sendo o valor médio posteriormente calculado. Foram utilizados os comprimentos de onda de 400 a $2.440 \mathrm{~nm}$, uma vez que as demais bandas apresentaram elevado grau de ruído, prejudicando a determinação dos modelos.

\section{Geração e calibração dos modelos}

As curvas espectrais foram pré-processadas, utilizando-se métodos consagrados em espectroscopia, visando corrigir possíveis ruídos, o que pode interferir na estabilidade da regressão. Primeiramente, realizouse a normalização do espectro com o processo de centralização em relação à média de reflectância das curvas. Posteriormente, para correção de possíveis erros por causa da não linearidade, os dados foram transformados utilizando-se a equação 2 :

$$
\log \frac{1}{R}
$$

em que: $\mathrm{R}=$ reflectância.

As novas curvas obtidas podem ser denominadas como absorbância aparente das amostras. As variações em razão do espalhamento da luz foram corrigidas utilizando a fórmula de variação normal padrão (SNV), para todos os atributos analisados (Viscarra Rossel, 2008).

Realizaram-se análises de componentes principais (ACP) para as curvas espectrais e atributos do solo, a fim de observar e eliminar possíveis outliers (Workman, 1992). Posteriormente, com o novo conjunto de dados, foram sorteados aleatoriamente $25 \%$ das curvas e seus respectivos atributos para posterior validação dos modelos que atingissem valores satisfatórios na fase de calibração.

Os modelos para predição dos atributos químicos e físicos avaliados foram gerados por meio da análise de regressão por mínimos quadrados parciais (PLSR), utilizando o software ParLes versão 3.1 (Viscarra Rossel, 2008). Esse método é comumente utilizado em espectroscopia, por eliminar de forma mais eficiente erros em razão da colinearidade entre variáveis, trabalhando com todos os comprimentos de onda, evitando dessa maneira a perda de informações importantes. O método PLSR é uma potente ferramenta de estimação do modelo de regressão linear, com base na decomposição das matrizes de variáveis resposta e de covariáveis (Martens \& Naes, 1989). O PLSR seleciona fatores ortogonais sucessivos com o objetivo de se obterem maiores covariâncias entre os dados preditores (x, neste caso as curvas espectrais) e as variáveis resposta (y, resultados das análises de laboratório).

$\mathrm{O}$ ajuste dos modelos PLSR procura encontrar o menor número de fatores que expliquem a maior parte da variação tanto das variáveis preditoras quanto das de resposta. Resumidamente, o conceito do método PLSR baseia-se em extrair componentes que consigam capturar as variâncias das covariáveis e também obter correlações com as variáveis independentes simultaneamente.

Geralmente, as matrizes de fatores resultantes têm menor dimensão que as matrizes x e y originais. Depois de gerados os modelos, a partir de novos espectros (x), a propriedade do solo (y) pode ser estimada com uma combinação (bi)linear dos fatores de $\mathrm{x}$ (Martens \& Naes, 1989).

Foi utilizado o método de validação cruzada denominado leave-one-out, sendo selecionados os modelos que apresentaram o menor número de fatores (NF), com maiores coeficientes de determinação $\left(\mathrm{R}^{2}\right)$, menores valores de raiz quadrada do erro médio (RMSE) definidos pela equação 3 e maiores valores de desvio-padrão do erro (RPD), definidos pela equação 4 (Williams, 2001): 


$$
\operatorname{RMSE}=\sqrt{\sum_{\mathrm{i}=1}^{\mathrm{n}-\mathrm{m}} \frac{(\mathrm{yi}-\mathrm{yi})}{\mathrm{n}-\mathrm{m}}}
$$

em que: $\mathrm{n}=$ número de amostras; $\mathrm{m}=$ número de amostras usadas para predição; e yi' - yi = valores preditos e observados, respectivamente.

$$
\mathrm{RPD}=\frac{\mathrm{sd}}{\mathrm{SEP}}
$$

em que: sd = taxa de desvio-padrão dos dados analisados; e SEP = erro-padrão da predição.

\section{Validação dos dados e avaliação dos modelos}

Quando os modelos gerados atingiram valores que permitiriam sua utilização na determinação de altas e baixas concentrações, segundo a literatura, do atributo do solo em questão, esses foram testados para verificar sua capacidade de estimar tais propriedades em $25 \%$ das amostras, diferentes daquelas utilizadas na calibração do modelo (Dardenne at al., 2000). Esse processo, segundo o autor, fornece uma ideia mais realista da exatidão dos modelos criados.

A avaliação do desempenho dos modelos foi realizada com base no trabalho de Araújo (2008), que utilizou os parâmetros propostos por Sayes et al. (2005) para indicar quatro faixas de satisfação para os valores de $R^{2}$, sendo essas: 0,50 e 0,65, com capacidade de descriminação entre altas e baixas concentrações do atributo; 0,66 a 0,81, modelos de predição aceitáveis; 0,82 a 0,90, modelos de predição bons; e acima de 0,90, excelentes. Com relação aos valores de RPD, Dunn et al. (2002) e Chang et al. (2001) sugeriram que valores menores que 1,5 sejam insuficientes para a maioria das aplicações; os entre 1,5 e 2,0, considerados úteis; e os acima de 2,0, excelentes.

\section{RESULTADOS E DISCUSSÃO}

\section{Caracterização das curvas espectrais de TFSE e ACC}

A resposta espectral para o NVef, tanto para amostras de TFSE como para ACC é apresentada na figura 1. As feições de absorções não sofreram grandes alterações entre os dois tipos de amostra, assim como a tendência horizontal e a baixa reflectância da curva espectral, típica dessa classe de solo (Epiphanio et al., 1992).

As ACC tiveram sua reflectância sempre menores que as de TFSE. Isso se deveu basicamente aos efeitos da umidade decorrente da forte absorção exercida pela molécula da água sobre a energia eletromagnética (Dalmolin et al., 2005) e da rugosidade de superfície, aumentando assim o espalhamento da luz refletida (Accioly \& Huete, 2000). A umidade média da ACC foi de $0,33 \mathrm{~cm}^{3} \mathrm{~cm}^{-3}$, enquanto na TFSE a água gravitacional foi quase totalmente removida pela secagem em estufa. Já a rugosidade foi bem menor na TFSE, uma vez que em sua preparação essa foi peneirada em malha de $2 \mathrm{~mm}$.

Também, em relação às ACC, é que a umidade das amostras promove a redução das feições de absorção de óxidos de Fe e o aumento das feições ligadas à molécula de água em $1.900 \mathrm{~nm}$, decorrente dos processos vibracionais do grupo $\mathrm{OH}$, tendo ainda as feições acima de $2.250 \mathrm{~nm}$ desaparecidas, em razão do efeito de ruídos aleatórios, que são mais acentuados nesses comprimentos de onda para superfícies com maior rugosidade (Gong \& Zang, 1999).

Para as amostras de TFSE, a intensidade de reflectância da camada de $0,0-0,2 \mathrm{~m}$ foi menor que a da camada de 0,2-0,4 m. Isso ocorreu principalmente por causa da presença de maiores teores de matéria orgânica (MO) na camada superficial, que para essas curvas espectrais foi em média de $1,93 \%$ na camada 0,0-0,2 m e de 1,56\%, na 0,2-0,4 m (Quadro 2). A MO reduziu a intensidade de reflectância para todas as faixas do espectro analisadas, sendo essa mais intensa em menores comprimentos de onda (Galvão \& Vitorello, 1998). Isso explica o fato que para comprimentos de onda maiores que $1.850 \mathrm{~nm}$ não são observadas grandes diferenças de intensidade de reflectância para as curvas espectrais dos solos de TFSE (Figura 1).

As ACC não evidenciaram o mesmo comportamento; a camada de 0,0-0,2 m refletiu sempre mais que a 0,2-0,4 m. Pode-se relacionar esse fato à maior umidade da camada superior. A umidade média das amostras que geraram as curvas espectrais de ACC foi de $0,29 \mathrm{~cm}^{3} \mathrm{~cm}^{-3}$ na camada superficial, enquanto no subsolo esses valores foram de 0,38 $\mathrm{cm}^{3} \mathrm{~cm}^{-3}$.

\section{Calibração dos modelos de predição}

A estatística descritiva dos resultados das análises químicas e físicas para todas as variáveis estudadas é apresentada no quadro 2 . Os modelos gerados para $\mathrm{P}$, $\mathrm{K}, \mathrm{Ca}, \mathrm{Mg}, \mathrm{SB}$ e $\mathrm{V} \%$, nas duas profundidades analisadas, não evidenciaram resultados satisfatórios segundo os critérios adotados para classificação dos modelos, com valores de $\mathrm{R}^{2}$ inferiores a 0,50 e $\mathrm{RPD}$ menores que 1,50, o que impossibilitou sua utilização. Uma das explicações para esse fato (com exceção do $\mathrm{K})$ foi a baixa variação dos teores desses elementos no solo, uma vez que a concentração deles na composição da vinhaça é pequena (Prada et al., 1998). Já para o íon $\mathrm{K}$, outros estudos observaram que sua estimativa usando a região do visível e o infravermelho próximo não é aceitável, principalmente se tratando de solos com alto teor de argila (Islam et al., 2003).

Os parâmetros utilizados na calibração dos modelos, bem como a sua capacidade em predizer os demais atributos em estudo nas profundidades avaliadas, são apresentados no quadro 3. Para os atributos $\mathrm{pH}, \mathrm{MO}, \mathrm{H}+\mathrm{Al}$ e CTC, os valores de $\mathrm{R}^{2}$ 


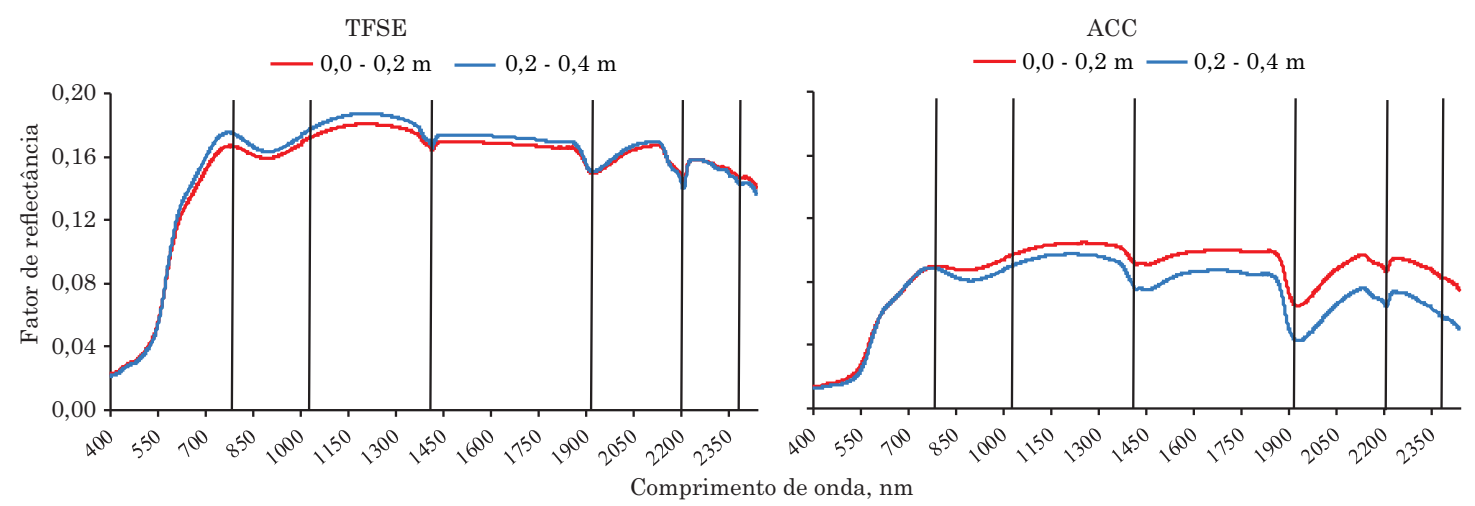

Figura 1. Curva espectral média para o NVef sem aplicação de vinhaça, nas profundidades de 0,0-0,2 e 0,2-0,4 m.

Quadro 2. Estatística descritiva das análises químicas e físicas do solo, nas profundidades em estudo

\begin{tabular}{|c|c|c|c|c|c|c|c|c|}
\hline Atributo & Mínimo & Máximo & Média & $\mathrm{S}^{2}$ & Mínimo & Máximo & Média & $\mathrm{S}^{2}$ \\
\hline & \multicolumn{4}{|c|}{$0,0-0,2 \mathrm{~m}$} & \multicolumn{4}{|c|}{$-0,2-0,4 \mathrm{~m}$} \\
\hline Argila $\left(\mathrm{g} \mathrm{kg}^{-1}\right)$ & 398,0 & 540,0 & 464,0 & 34,0 & 415,0 & 548,0 & 484,3 & 34,5 \\
\hline Silte $\left(\mathrm{g} \mathrm{kg}^{-1}\right)$ & 200,0 & 316,0 & 270,2 & 31,1 & 210,0 & 337,0 & 274,0 & 29,4 \\
\hline Areia $\left(\mathrm{g} \mathrm{kg}^{-1}\right)$ & 230,0 & 300,0 & 265,8 & 16,9 & 210,0 & 290,0 & 241,6 & 20,4 \\
\hline $\mathrm{pH}\left(\mathrm{CaCl}_{2}\right)$ & 4,2 & 5,7 & 4,8 & 0,3 & 4,2 & 5,8 & 5,1 & 0,3 \\
\hline $\mathrm{MO}\left(\mathrm{g} \mathrm{kg}^{-1}\right)$ & 6,0 & 27,0 & 19,3 & 3,0 & 9,0 & 29,0 & 15,6 & 3,3 \\
\hline $\mathrm{P}\left(\mathrm{mg} \mathrm{dm}^{-3}\right)$ & 3,0 & 31,0 & 9,3 & 5,4 & 3,0 & 14,0 & 6,9 & 2,2 \\
\hline $\mathrm{K}\left(\mathrm{mmol}_{\mathrm{c}} \mathrm{dm}^{-3}\right)$ & 0,5 & 6,0 & 3,3 & 1,0 & 0,5 & 11,8 & 2,5 & 1,7 \\
\hline $\mathrm{Ca}\left(\mathrm{mmol}_{\mathrm{c}} \mathrm{dm}^{-3}\right)$ & 18,0 & 45,0 & 33,0 & 5,0 & 16,0 & 47,0 & 32,4 & 5,1 \\
\hline $\mathrm{Mg}\left(\mathrm{mmol}_{\mathrm{c}} \mathrm{dm}^{-3}\right)$ & 7,0 & 21,0 & 14,4 & 2,9 & 5,0 & 23,0 & 15,4 & 3,2 \\
\hline $\mathrm{H}+\mathrm{Al}\left(\mathrm{mmol}_{\mathrm{c}} \mathrm{dm}^{-3}\right)$ & 20,0 & 64,0 & 36,6 & 6,9 & 18,0 & 80,0 & 30,6 & 8,2 \\
\hline $\mathrm{SB}\left(\mathrm{mmol}_{\mathrm{c}} \mathrm{dm}^{-3}\right)$ & 28,0 & 68,0 & 50,8 & 7,4 & 24,0 & 71,0 & 50,5 & 7,5 \\
\hline $\mathrm{CTC}_{\mathrm{pH} 7}\left(\mathrm{mmol}_{\mathrm{c}} \mathrm{dm}^{-3}\right)$ & 53,0 & 102,0 & 87,4 & 7,5 & 64,0 & 107,0 & 80,9 & 7,9 \\
\hline $\mathrm{V}(\%)$ & 37,0 & 75,0 & 58,1 & 6,9 & 23,0 & 77,0 & 62,3 & 8,1 \\
\hline
\end{tabular}

Quadro 3. Resultado da calibração dos modelos PLS, nas profundidades de 0,0-0,2 e 0,2-0,4 m, para terra fina seca em estufa (TFSE) e amostra em condição de campo (ACC)

\begin{tabular}{|c|c|c|c|c|c|c|c|c|}
\hline \multirow{2}{*}{ Atributo } & \multicolumn{4}{|c|}{ TFSE } & \multicolumn{4}{|c|}{$\mathrm{ACC}$} \\
\hline & NF & RMSE & RPD & $\mathbf{R}^{2}$ & NF & RMSE & RPD & $\mathbf{R}^{2}$ \\
\hline & \multicolumn{8}{|c|}{$0,0-0,2 \mathrm{~m}$} \\
\hline Argila $\left(\mathrm{g} \mathrm{kg}^{-1}\right)$ & 16 & 7,67 & 4,44 & 0,96 & 13 & 8,34 & 4,28 & 0,95 \\
\hline Silte $\left(\mathrm{g} \mathrm{kg}^{-1}\right)$ & 15 & 19,65 & 1,58 & 0,59 & 11 & 21,89 & 1,42 & 0,50 \\
\hline Areia $\left(\mathrm{g} \mathrm{kg}^{-1}\right)$ & 15 & 5,79 & 2,92 & 0,88 & 13 & 4,23 & 3,67 & 0,94 \\
\hline $\mathrm{pH}\left(\mathrm{CaCl}_{2}\right)$ & 10 & 0,37 & 1,51 & $0,55^{*}$ & 5 & 0,37 & 1,27 & $0,39^{*}$ \\
\hline $\mathrm{MO}\left(\mathrm{g} \mathrm{kg}^{-1}\right)$ & 11 & 3,82 & 1,67 & 0,64 & 6 & 3,14 & 1,63 & 0,62 \\
\hline $\mathrm{H}+\mathrm{Al}\left(\mathrm{mmol}_{\mathrm{c}} \mathrm{dm}^{-3}\right)$ & 13 & 7,37 & 1,56 & $0,59 *$ & 6 & 5,82 & 1,36 & $0,46^{*}$ \\
\hline \multirow[t]{2}{*}{$\mathrm{CTC}_{\mathrm{pH} 7}\left(\mathrm{mmol}_{\mathrm{c}} \mathrm{dm}^{-3}\right)$} & 12 & 8,07 & 1,69 & $0,65^{*}$ & 6 & 10,72 & 1,27 & $0,40^{*}$ \\
\hline & \multicolumn{8}{|c|}{$0,2-0,4 \mathrm{~m}$} \\
\hline Argila $\left(\mathrm{g} \mathrm{kg}^{-1}\right)$ & 16 & 13,12 & 2,63 & 0,87 & 13 & 13,41 & 2,58 & 0,85 \\
\hline Silte $\left(\mathrm{g} \mathrm{kg}^{-1}\right)$ & 15 & 23,21 & 1,27 & 0,33 & 11 & 22,52 & 1,30 & 0,36 \\
\hline Areia $\left(\mathrm{g} \mathrm{kg}^{-1}\right)$ & 15 & 6,70 & 3,03 & 0,88 & 13 & 7,88 & 2,58 & 0,86 \\
\hline $\mathrm{pH}\left(\mathrm{CaCl}_{2}\right)$ & 14 & 0,29 & 1,43 & 0,52 & 7 & 0,30 & 1,48 & 0,55 \\
\hline MO $\left(\mathrm{g} \mathrm{kg}^{-1}\right)$ & 13 & 2,30 & 1,67 & 0,64 & 7 & 2,59 & 1,46 & 0,54 \\
\hline $\mathrm{H}+\mathrm{Al}\left(\mathrm{mmol}_{\mathrm{c}} \mathrm{dm}^{-3}\right)$ & 15 & 4,83 & 1,46 & 0,51 & 10 & 4,34 & 1,43 & 0,52 \\
\hline $\mathrm{CTC}_{\mathrm{pH} 7}\left(\mathrm{mmol}_{\mathrm{c}} \mathrm{dm}^{-3}\right)$ & 12 & 5,55 & 1,59 & 0,61 & 6 & 6,47 & 1,49 & 0,55 \\
\hline
\end{tabular}

NF: número de fatores; RMSE: raiz quadrada do erro médio; RPD: desvio-padrão do erro: $\mathrm{R}^{2}$ : coeficiente de determinação; * diferença significativa pelo teste $t$ de Student $(p<0,05)$. 
estiveram entre 0,50 e 0,65 para as amostras de TFSE; seus RPD estiveram muito próximos ou acima de 1,5 , podendo então esses modelos serem utilizados apenas para a distinção entre altas e baixas concentrações (Chang et al., 2001; Dunn et al., 2002; Sayes et al., 2005). Quando utilizadas as ACC, os valores de $\mathrm{R}^{2}$ ficaram abaixo de 0,50 para $\mathrm{pH}, \mathrm{H}+\mathrm{Al} \mathrm{e}$ CTC, na camada de 0,0-0,2 m, e silte, na de $0,2-0,4 \mathrm{~m}$ (Quadro 3).

Ainda sobre esses dados, na camada superficial, apenas o modelo para MO não evidenciou diferença estatística entre os dois tipos de amostras. Para a camada de 0,2-0,4 m, todos os modelos foram estatisticamente iguais, quando comparados pelo teste t a $5 \%$.

Para a granulometria do solo, foram gerados modelos considerados de "bons" a "excelentes", com relação à exatidão de predição. Na fase de calibração para a camada de 0,0-0,2 m, a fração argila obteve valores de $\mathrm{R}^{2}=0,96$ e $\mathrm{RPD}=4,44$ para TFSE; e $\mathrm{R}^{2}=$ 0,96 e $\mathrm{RPD}=4,44$ para $\mathrm{ACC}$, o mesmo ocorrendo com a fração areia, que alcançou $\mathrm{R}^{2}=0,88$ e $\mathrm{RPD}=2,92$ para TFSE e $\mathrm{R}^{2}=0,94$ e RPD $=3,67$ para ACC. Os modelos para silte apenas permitiram a determinação de alta ou baixa concentração. No entanto, o teor dessa fração pode ser obtido pela diferença entre o percentual de areia+argila (Quadro 3). Para granulometria, não houve diferença estatística entre os modelos de ACC e TFSE.

Em um estudo realizado com o objetivo de definir as propriedades para quatro classes de solos brasileiros, Fiorio \& Demattê (2009) encontraram valores um pouco inferiores de $\mathrm{R}^{2}$ para modelos de determinação de argila e areia, sendo esses de 0,86 e 0,81 , respectivamente. No entanto, ressalta-se que esses autores utilizaram análise de regressão múltipla e propuseram uma equação global para avaliação desses atributos para diferentes solos e horizontes.

\section{Validação dos modelos de predição}

Os modelos para determinação de $\mathrm{pH}, \mathrm{MO}, \mathrm{H}+\mathrm{Al}$, CTC e as frações areia, silte e argila foram testados para amostras independentes não utilizadas na fase de calibração, nas duas camadas de solo em estudo, tanto para TFSE como ACC (Figuras 2, 3, 4 e 5).

Os modelos para as ACC foram testados mesmo quando os valores de calibração foram baixos, desde que os modelos para TFSE tenham alcançado valores significativos, uma vez que o objetivo deste trabalho foi comparar a capacidade de predição de atributos do solo a partir de dois tipos de amostras. Não houve casos em que a calibração foi satisfatória para as ACC, bem como para TFSE.

Para a granulometria do solo na profundidade de 0,0-0,2 m (Figuras 2 e 3), os modelos tiveram bom desempenho na predição de teores em novas amostras de solos; os resultados para areia $\left(\mathrm{R}^{2}>0,85 \mathrm{e}\right.$ $\mathrm{RPD}>2,0$ ) tiveram melhores resultados que para argila $\left(0,70<R^{2}>0,85\right.$ e $\left.1,8<R P D>2,0\right)$, sendo esses resultados considerados de aceitáveis a bons. Já para o teor de silte, observou-se a capacidade apenas de discriminação entre altas e baixas concentrações, tanto nas amostras de TFSE como nas ACC. Utilizando o mesmo método e faixa do espectro, Cozzolino \& Moron (2003) obtiveram valores semelhantes ao deste estudo.

Para os atributos químicos $\mathrm{pH}, \mathrm{MO}, \mathrm{H}+\mathrm{Al}$ e CTC, as amostras de TFSE (Figura 2) indicaram potencial apenas para diferenciação de baixas e altas concentrações desses elementos $\left(0,50<R^{2}>0,65\right.$ e $\mathrm{RPD} \approx 1,5)$. No entanto, para as ACC (Figura 3) da camada superficial do solo, apenas os teores de MO evidenciaram resultados significativos. A baixa eficiência dos modelos para predição dos atributos químicos do solo observada neste trabalho pode estar ligada principalmente à característica do solo em estudo, que possui altos teores de argila, sendo sua curva espectral governada pela presença de óxidos, possuindo geralmente elevados teores de $\mathrm{MO}$, o que vem a mascarar os efeitos dos demais constituintes (Bellinaso et al., 2010).

Na camada de 0,2-0,4 m, a exatidão dos modelos para determinação da granulometria foi muito próxima à obtida na camada superficial do solo, sendo os modelos classificados como aceitáveis a bons. $\mathrm{Na}$ definição dos atributos químicos, os resultados também se aproximaram daqueles anteriormente citados para as amostras de TFSE (Figura 4). No entanto, o desempenho das ACC (Figura 5) foi melhor quando comparado ao resultado da camada superior, se aproximando dos obtidos com TFSE.

Segundo Zanette et al. (2007), a camada superficial do solo sofre maior influência externa, principalmente em relação à umidade, que geralmente apresenta alta variação espacial, sendo esse fenômeno normalizado nas subcamadas. Esse fato pode explicar o melhor desempenho das ACC na camada de 0,2-0,4 m.

\section{CONCLUSÕES}

1. As amostras terra fina seca em estufa (TFSE) apresentaram maior intensidade de reflectância e feições de absorção mais definidas quando comparadas com as amostras em condição de campo (ACC), sendo as variações da primeira governadas principalmente pela MO, enquanto as da segunda pela umidade, que é o principal fator.

2. As frações granulométricas do solo foram estimadas com exatidão de aceitável a boa, tanto para amostras ACC quanto TFSE.

3. A determinação dos atributos químicos nas condições experimentais a que o solo foi submetido satisfez apenas a possibilidade de distinção entre altas e baixas concentrações de $\mathrm{pH}, \mathrm{MO}, \mathrm{H}+\mathrm{Al}$ e CTC. 

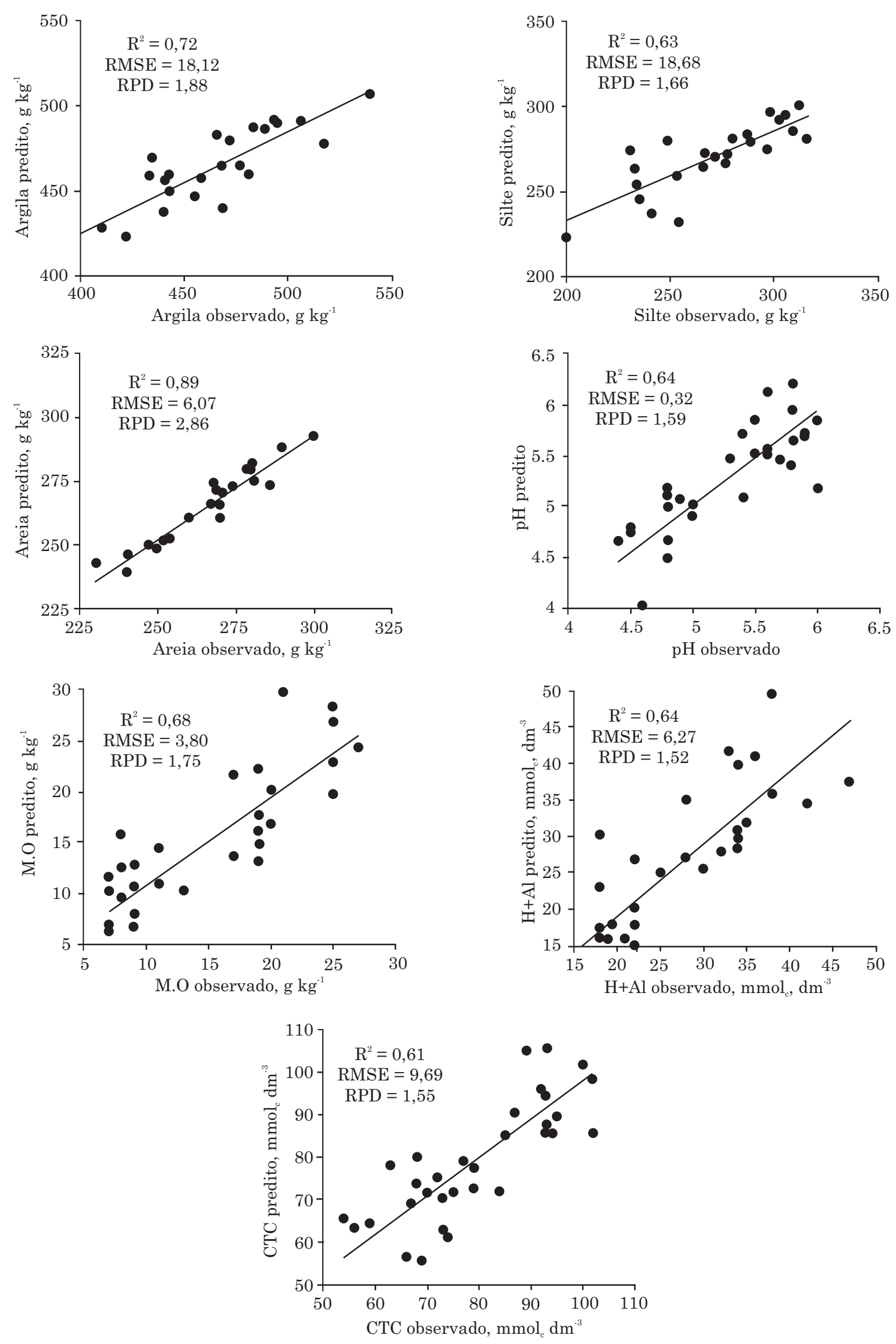

Figura 2. Validação dos modelos PLS para os atributos químicos e físicos, na profundidade de 0,0-0,2 m para TFSE. 

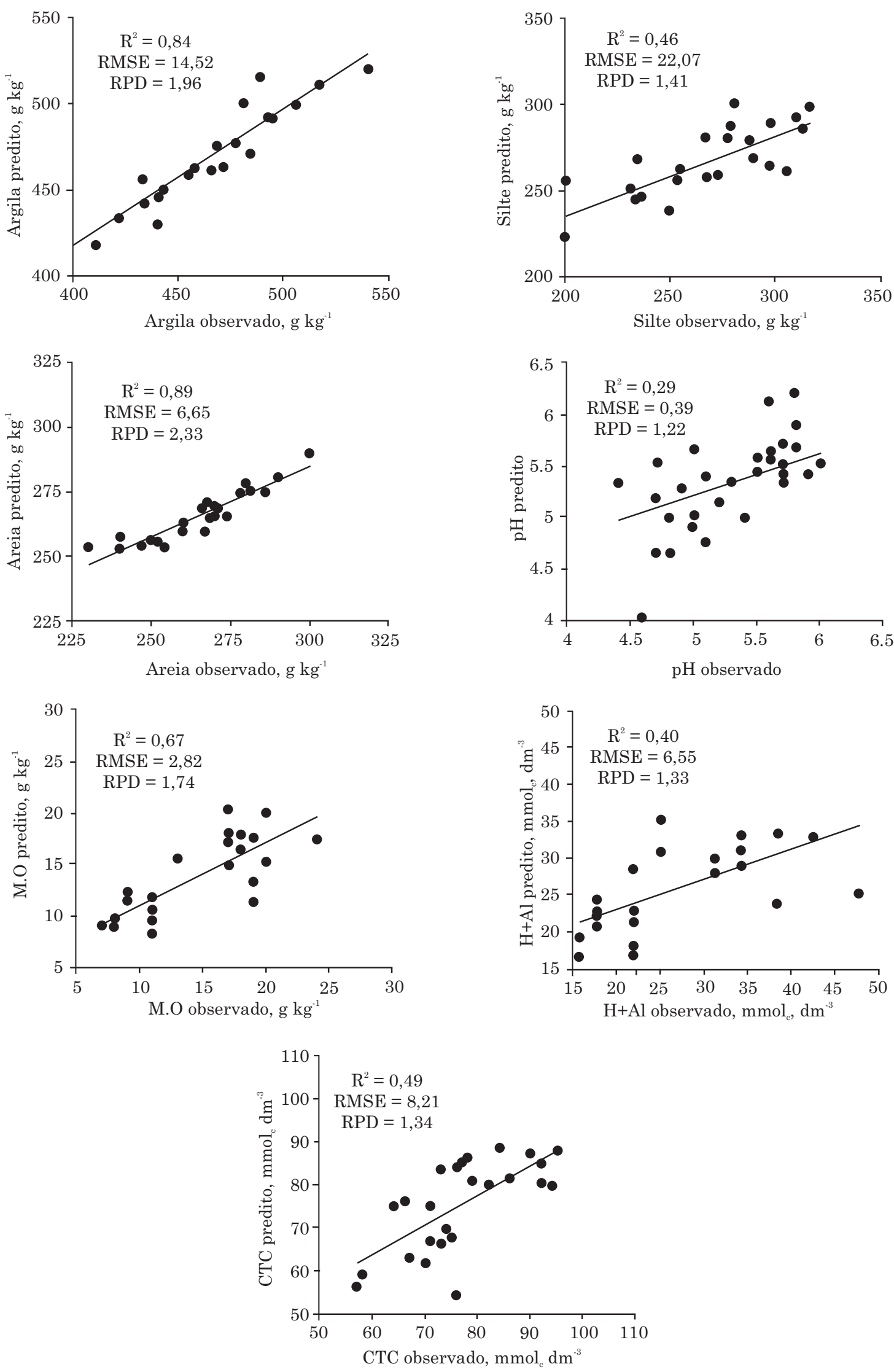

Figura 3. Validação dos modelos PLS para os atributos químicos e físicos, na profundidade de 0,0-0,2 m para ACC. 

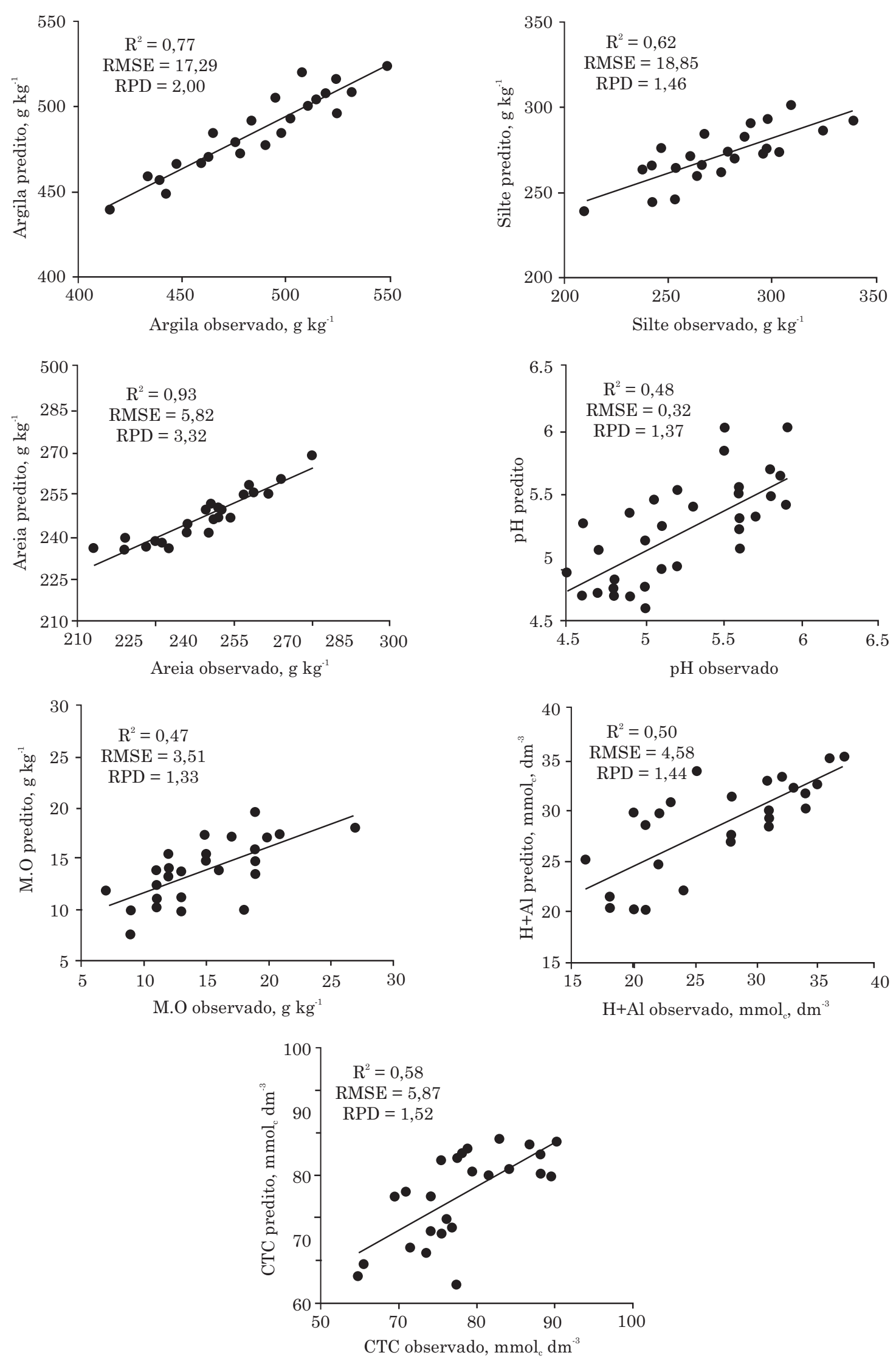

Figura 4. Validação dos modelos PLS para os atributos químicos e físicos, na profundidade de 0,2-0,4 $\mathrm{m}$ para TFSE. 

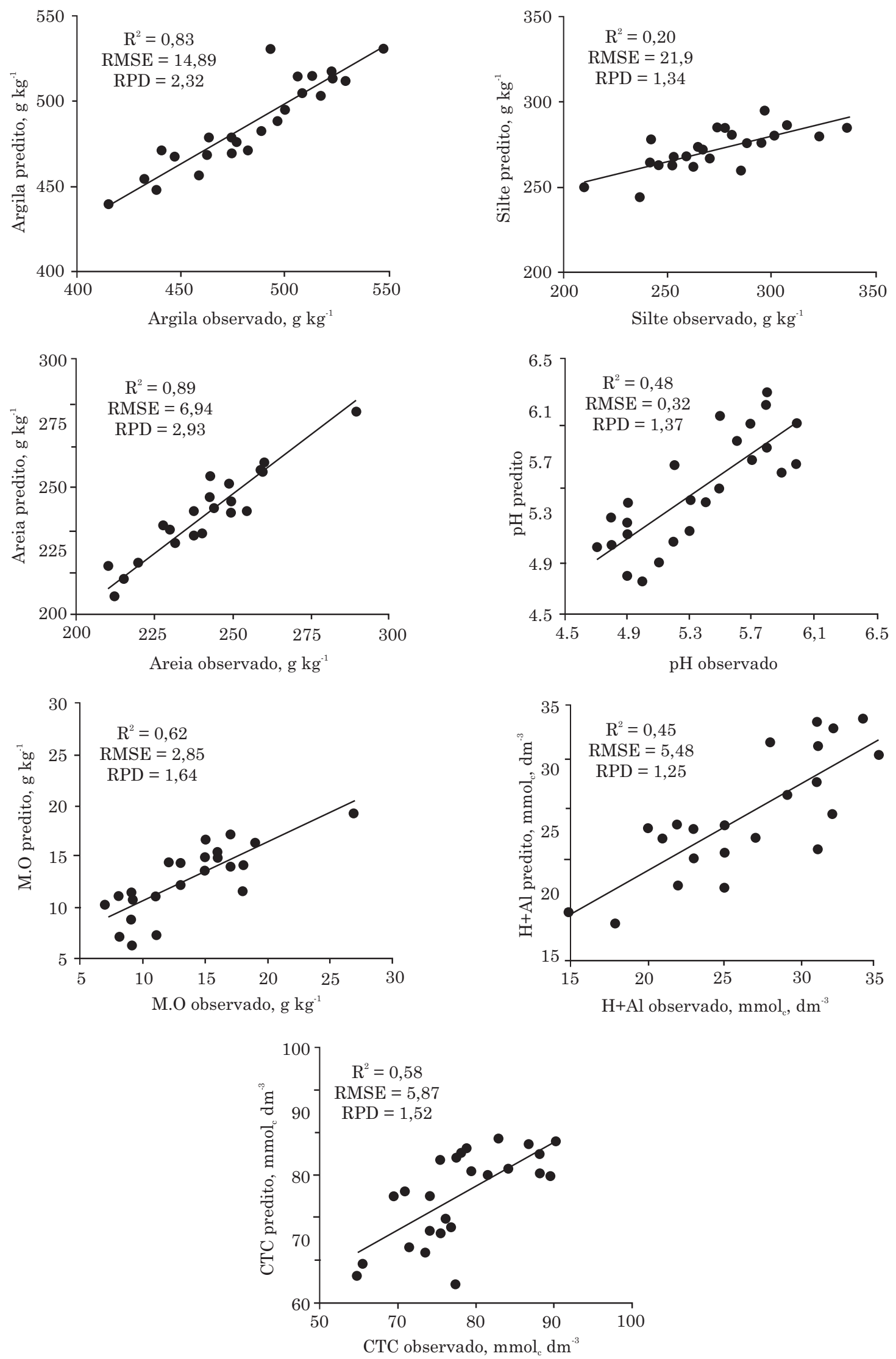

Figura 5. Validação dos modelos PLS para os atributos químicos e físicos, na profundidade de 0,2-0,4 $\mathrm{m}$ para ACC. 


\section{AGRADECIMENTOS}

À FAPESP, pela concessão do auxílio à pesquisa (Processo n ${ }^{\circ}$ 2010/16368-9 e 2010/00787-2) e aquisição do aparelho Hiperespectral FieldSpec (Processo $\mathrm{n}^{\circ}$ 2007-54976-8). Ao CNPq, pela concessão da bolsa de mestrado ao primeiro autor (Processo n ${ }^{\circ}$ 130945/2010-2).

\section{LITERATURA CITADA}

ACCIOLY, L.J.O. \& HUETE, A.R. Resposta espectral de solos em razão do ângulo de visada, da umidade e da rugosidade superficial. Pesq. Agropec. Bras., 35:2473-2484, 2000.

ARAÚJO, S.R. Sensoriamento remoto laboratorial na detecção de alterações químicas no solo pela aplicação de corretivos. Piracicaba, Escola Superior de Agricultura "Luiz de Queiroz", 2008. 130p. (Dissertação de Mestrado)

BELliNASO, H.; DEMATTÊ, J.A.M. \& ROMEIRO, S.A. Spectral library and its use in soil classification. R. Bras. Ci. Solo, 34:861-867, 2010.

BROWN, D.J.; SHEPERD, K.D.; WLASH, M.G.; MAYS, M.D. \& REINSCH, T.G. Global soil characterization with VNIR diffuse reflectance spectroscopy. Geoderma, 132:273-290, 2006.

CAMARGO, O.A.; MONIZ, A.C.; JORGE, J.A. \& VALADARES, J.M. Métodos de análise química, mineralógica e física de solos do IAC. Campinas, Instituto Agronômico de Campinas, 1986. 94p. (Boletim Técnico, 106)

CHANG, C.W.; LAIRD, D.A.; MAUSBACH, M.J. \& HURBURGH, J.C.R. Near-infrared reflectance spectroscopy principal components regression analysis of soil properties. Soil Sci. Soc. Am. J., 65:480-490, 2001.

COHEN, M.; MYLAVARAPU, R.S.; BOGREKCI, I.; LEE, W.S. \& CLARK, M.W. Reflectance spectroscopy for routine agronomic soil analyses. Soil Sci. Soc. Am. J., 172:469485, 2007.

COMPANHIA DE TECNOLOGIA DE SANEAMENTO AMBIENTAL - CETESB. Norma técnica P4.231. São Paulo, 2005. 17p.

COZZOLINO, D. \& MORON, A. The potential of near-infrared reflectance spectroscopy to analyze soil chemical and physical characteristics. J. Agric. Sci., 140:65-71, 2003.

DALMOLIN, R.S.D.; GONÇALVES, C.N. \& DICK, D.P. Relação entre os constituintes do solo e seu comportamento espectral. Ci. Rural, 35:481-489, 2005.

DARDENNE, P.; SINNAEVE, G. \& BAETEN, V. Multivariate calibration and chemometrics for near infrared spectroscopy: Which method?. J. Near Infrared Spec., 8:229-237, 2000 .

DEMATTÊ, J.A.M. \& GARCIA, G.J. Avaliação de atributos de Latossolo Bruno e de Terra Bruna Estruturada da região de Guarapuava, Paraná, por meio de sua energia refletida. R. Bras. Ci. Solo, 23:343-355, 1999.
DEMATTÊ, J.A.M.; NANNI, M.R.; SILVA, A.P.; MELO FILHO, J.F.; SANTOS, W.C. \& CAMPOS, R.C. Soil density evaluated by spectral reflectance as an evidence of compaction effects. Inter. J. Remote Sens., 31:403-422, 2010.

DUNN, B.W.; BEECHER, H.G.; BATTEN, G.D. \& CIAVARELLA, S. The potential of near-infrared reflectance spectroscopy for soil analysis - A case study from the Riverine plain of south eastern Australia. Aust. J. Exp. Agric., 42:607-614, 2002.

EMPRESA BRASILEIRA DE PESQUISA AGROPECUÁRIA - EMBRAPA. Sistema brasileiro de classificação de solos. Rio de Janeiro, 2006. 306p.

EPIPHANIO, J.C.N.; FORMAGGIO, A.R.; VALVFRIANO, M. \& OLIVEIRA, J.B. Comportamento espectral de solos do Estado de São Paulo. São José dos Campos, INPE, 1992. $131 \mathrm{p}$.

FIORIO, P.R. \& DEMATTÊ, J.A.M. Orbital and laboratory spectral data to optimize soil analysis. Sci. Agric., 66:250257, 2009.

GALVÃO, L.S. \& VITORELLO, I. Role of organic matter in obliterating the effects of iron on spectral reflectance and color of Brazilian tropical soils. Inter. J. Remote Sens., 19:1969-1979, 1998.

GONG, P. \& ZHANG, A. Noise effect on linear stectral unmixing. Lect. Notes. Comput. Sci., 5:52-57, 1999.

HENDERSON, T.L.; BAUMGARDNER, M.F.; FRANZMEIER, D.P.; STOTT, D.E. \& COSTER, D.C. High dimensional reflectance analysis of soil organic matter. Soil Sci. Soc. Am. J., 56:865-872, 1992.

ISLAM, K.; SINGH, B. \& McBRATNEY, A.B. Simultaneous estimation of various soil properties by ultra-violet, visible and near-infrared reflectance spectroscopy. Aust. J. Soil Res., 41:1101-1114, 2003.

LEE, W.S.; SANCHEZ, T.F.; MYLAVARAPU, R.S. \& CHOE, J.S. Estimating chemical properties of Florida soils using spectral reflectance. Am. Soc. Agric. Eng., 46:1443-1453, 2003.

MARTENS, H. \& NAES, T. Multivariate calibration. Chichester, John Wiley, 1989. 419p.

PRADA, S.M.; GUEKEZIAN, M. \& SUÁREZ-ILHA, M.E.V. Metodologia analítica para a determinação de sulfato em vinhoto. Quím. Nova, 21:249-252, 1998.

RAIJ, B.van \& QUAGGIO, J.A. Métodos de análise do solo para fins de fertilidade. Campinas, Instituto Agronômico de Campinas, 1983. 40p. (IAC. Boletim Técnico, 81)

SAYES, W.; MOUAZEM, A.M. \& RAMON, H. Potential for onsite and online analyses of pig manure using visible and near infrared reflectance spectroscopy. Biosyst. Eng., 91:393-402, 2005.

SILVA, M.A.S.; GRIEBELER, N.P. \& BORGES, L.C. Uso de vinhaça e impactos nas propriedades do solo e lençol freático. R. Bras. Eng. Agríc. Amb., 11:108-114, 2007. 
VISCARRA ROSSEL, R.A. ParLeS: Software for chemometric analysis of spectroscopic data. Chemometr. Intell. Lab., 90:72-83, 2008.

VISCARRA ROSSEL, R.A.; CATTLE, S.R.; ORTEGA, A. \& FOUAD, Y. In situ measurements of soil colour, mineral composition and clay content by Vis-NIR spectroscopy. Geoderma, 150:253-266, 2009.

VISCARRA ROSSEL, R.; RIZZO, R.; DEMATTÊ, J.A.M. \& BEHRENS, T. Spatial modeling of a soil fertility index using visible-near-infrared spectra and terrain attributes. Soil Sci. Soc. Am. J., 74:1293-1300, 2010.
WILLIAMS, P.C. Implementation of near-infrared technology. In: WILLIAMS, P. \& NORRIS, K., eds. Near-infrared technology in the agricultural and food industries. Saint Paul, American Association of Cereal Chemists, 2001. p.145-169.

WORKMAN, J.J. NIR spectroscopy calibrations basics. In: BURNS, D.A. \& CIUREZAK, E.W., eds. Handbook of near infrared analysis. New York, Edgard Blucher, 1992. p.247-280.

ZANETTE, S.V.; SAMPAIO, S.C.; SILVESTRE, M.G.; BOAS, M.A.V.; URIBE-OPAZO, M.A. \& QUEIROZ, M.M.F. Análise espacial da umidade do solo cultivado com soja sob dois sistemas de manejo. R. Bras. Eng. Agric. Amb., 11:239-247, 2007. 